\title{
Resistance Reduction by Stern-End-Bulb
}

\author{
(Second Report) \\ by Hideaki Miyata*, Member Yoshihiro Tsuchiya* \\ Takao Inui**, Member
}

\begin{abstract}
Summary
The characteristics of the waves in the vicinity of the stern and the mechanism of wave resistance reduction by stern-end-bulb are experimentally investigated. Observation of stern waves, measurement of disturbance velocities, wave height, momentum loss and pressure on hull surface are carried out, and they show that waves in the vicinity of the stern have nonlinear properties and that the effect of stern-end-bulb considerably consists in the attenuation of nonlinear stern waves. The problems of scale effect in wave making and wave reduction by stern-end-bulb and those of the design for the improved stern-end-bulb are also studied.
\end{abstract}

\section{Introduction}

Stern-End-Bulb (abbreviated as SEB hereafter) was developed by the authors in $1978^{1)}$ and its availability and effectiveness were demonstrated in the previous paper ${ }^{2}$. SEBs whose volumes were rather small could reduce wave resistance to a considerable degree. The ratio of EHP saving reached up to $5 \sim 6 \%$.

In the previous paper the method of designing SEB which is composed of the method of solving a simplified Neumann-Kelvin problem is proposed and it is shown that this method is useful especially for the decision of the optimal width of SEB for a container carrier and a passenger boat. However, the wave system in the vicinity of the stern is not that of linear dispersive waves, but it is of the nonlinear waves similar to free surface shock waves)5) (abbreviated as FSSW hereafter). SEB reduces FSSWs and linear dispersive waves simultaneously. Therefore, the characteristics of the waves must be carefully investigated for the improvement of the design procedure of SEB and for the clarification of the mechanism of wave resistance reduction.

This has been also the case for the bulbous bow developed about 20 years ago. The effect of bulbous bow has been explained by linear

\footnotetext{
* Department of Naval Architecture, The University of Tokyo

** Tamagawa University
}

wave making theories as an effect of wave cancellation. However, experimental investigations into the characteristics of waves have come to show us that a bulbous bow usually reduces FSSWs around the bow as well as linear waves measured as wave pattern resistance in the far-field ${ }^{6}$.

Chapters 2 and 3 are focused on the characteristics of stern waves of ships and the mechanism of wave reduction by SEB. Photographing wave pictures, measurements of disturbance velocities, wave height, momentum loss far behind and pressure distributions on the hull surface are undertaken with the models in Table 1. In Chapter 4 the scale effect in the wave height and in the effectiveness of SEB is studied, and in Chapter 5 improved design of SEB for a passenger boat is discussed.

\section{Characteristics of waves in the vicinity of stern}

\subsection{Wave picture}

Waves from the aftbody of a ship are sometimes very conspicuous and it is intuitively understood that they contribute to the resistance of ships to a great degree. The wave pictures of full-scale passenger boats in Fig. 1 of ref. 2) and Fig. 21 of this paper show intense stern waves. It is naturally recognized that more attention and effort should be devoted to the stern waves in the near-field of ships.

Wave pattern pictures of a fine deep-drafted ship model are present in Fig. 1 which were 
Table 1 Principal particulars of tested models

\begin{tabular}{|c|c|c|c|c|c|c|}
\hline$(\mathrm{m})$ & $L_{p p}$ & $B$ & $d$ & $C_{B}$ & trim & \\
\hline MB1 & dlu ${ }_{2.500}$ & tro.3054 & 0.400 & 0.5810 & fistolas? & wall-sided \\
\hline rudder model & 0.300 & 0.048 & $0: 100$ & & 0 & \\
\hline SR138 (2.5) & 2.500 & 0.3846 & 0.088 & 0.524 & $1.0 \%$ & trial condition \\
\hline
\end{tabular}

for other models see ref, 2)

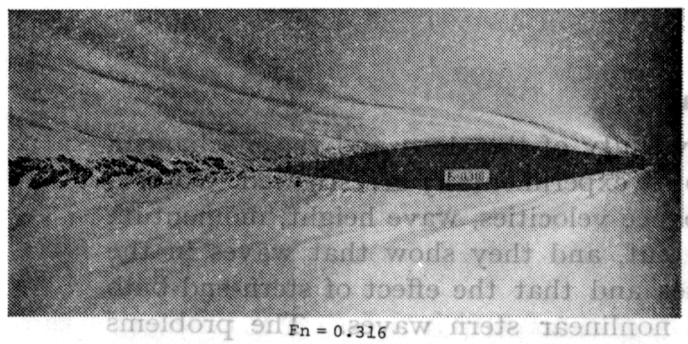

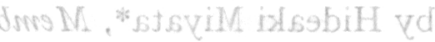
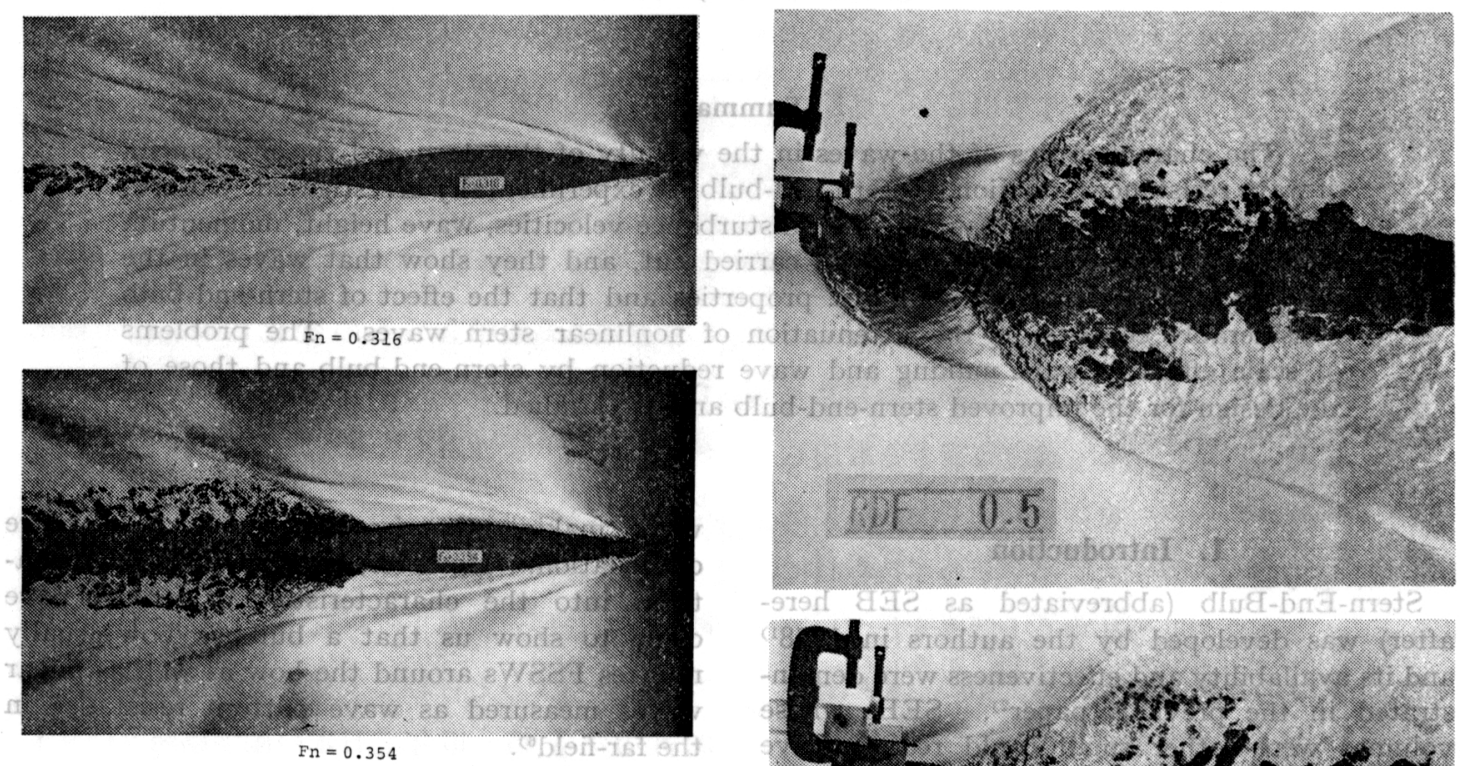

Fig. 1 Stern wave picture of MB1 at $F n=$ 0.316 and 0.354

photographed by our routine technique with aluminum powder film on the water surface. At $F n=0.316$ the waves from the after end of the model seem to be different from linear Kelvin waves, though their scale is not great. But, the waves appear in an astonishingly peculiar manner when the speed of advance is increased up to $F n=0.354$. The stern waves at $F n=0.354$ generate lines of discontinuity across which aluminum powder cannot pass through. $W$ The existence of the black region behind the wave front implies the generation of the lines of discontinuity, and the free surface in this region seems to have some turbulence. These qualitative properties are the same with FSSWs around the bow ${ }^{5}$ ?

More exaggerated cases are shown in Fig. 2. The model is rudder-shaped which is roundnosed and sharp-ended. The picture of this model towed at $F n=0.5$ shows that a normal FSSW which is analogous to the normal shock

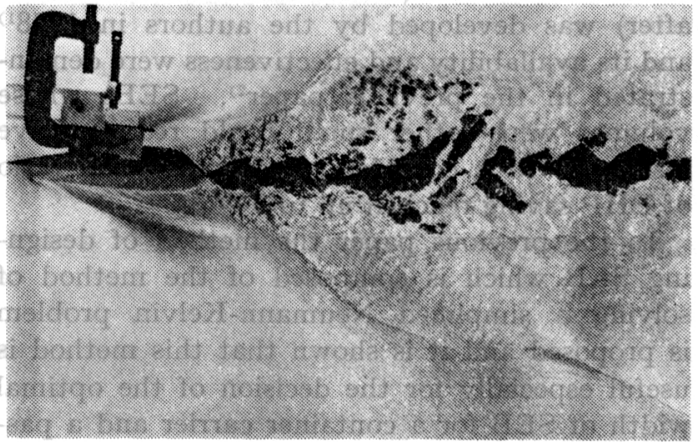

Fig. 2 Stern wave picture of rudder model at $F n=0.5$, round nosed (above) and sharp nosed (below)

wave in supersonic flow is generated around round-nosed bow and that the other great peculiar wave is generated from the sharp-ended stern. In the case when it is towed in the reverse condition the bow wave is of oblique type because of the sharpness of the entrance and the waves from the stern are of a different form. With these pictures we can conclude that both bow and stern waves in the near-field are nonlinear ones which constitute the lines of discontinuity and that the nonlinear waves at the stern, as will be further discussed in the following chapters, have much more complicated 
erties.dop than the nonlinear waves at the bow that have been systematically explained in an arralogous manner by the author ${ }^{7}$.

The change of wave formation at the stern of SR $138 \times 2$ is present in Fig. 3. The formation of the waves is considerably changed with the increase of advance speed as if a critical speed

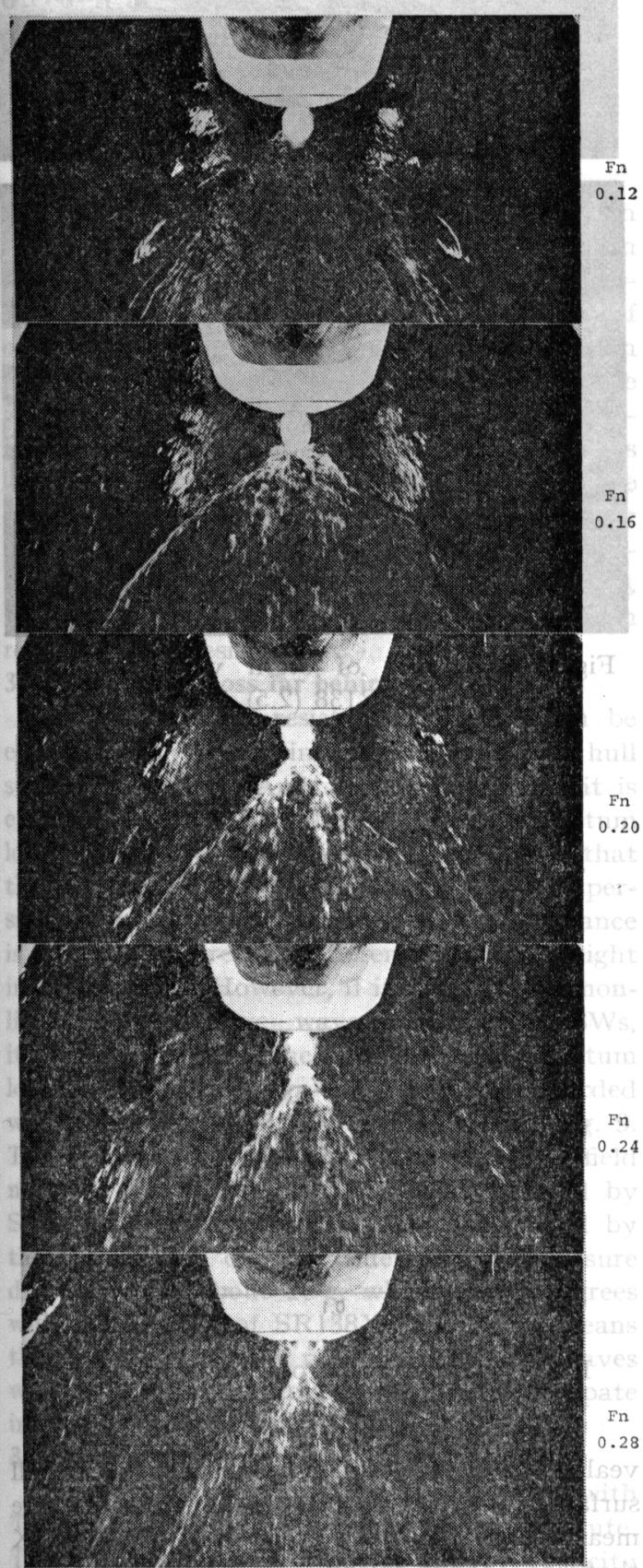

Fig. 3 Change of stern wave formation on jofistánil SR 138X2 (10) exists. Until $F n=0.16$ the wave is of normal type and it is transformed into oblique type at $F_{n}=0.20$. Then, the angle of the oblique nonlinear wave to the centerline of the ship model (shock angle) decreases with the increase of advance speed. In the field of supersonic aerodynamics it is well known that a normal shock wave is a strong shock that produces large energy loss and an oblique shock is a comparatively weak shock that produces smaller energy loss. This is also the case in the problem of FSSW, which is demonstrated in Figs. 18, 20 and 24 of ref. 4 ) in which the transition from normal to oblique renders abrupt decrease of the resistance due to FSSW. The change of shock angle of oblique FSSW is also intimately related to the change of the resistance due to FSSW as can be seen in Fig. 32 of ref. 6). The normal-type wave at $F n=0.16$ in Fig. 3 does not appear when SEB is not equipped and consequently the wave resistance of $\mathrm{SR} 138 \times 2$ in the speed range below $F n=0.20$ is greater than SR138, whereas wave pattern resistance is rather small as is seen in Figs. 11 and 12. In other words SR138X2 produces a small hump around $F \boldsymbol{n}=0.17$ where stern waves of normal type are present, and with their transition into oblique type, wave resistance experiences a little abrupt decrease. The change of wave formation and its contribution to resistance are very analogous to those of FSSWs around the bow and shock waves in supersonic flow.

\subsection{Disturbance velocities}

Disturbance velocities in the vertical plane behind the ship model SR138 are measured with a five-hole pitot tube in order to clarify the properties of nonlinear waves from the stern that are visualized in Fig. 17 of ref. 1). The recorded transverse wave profile is not (linearly) wavy but stepped with steep slope along the
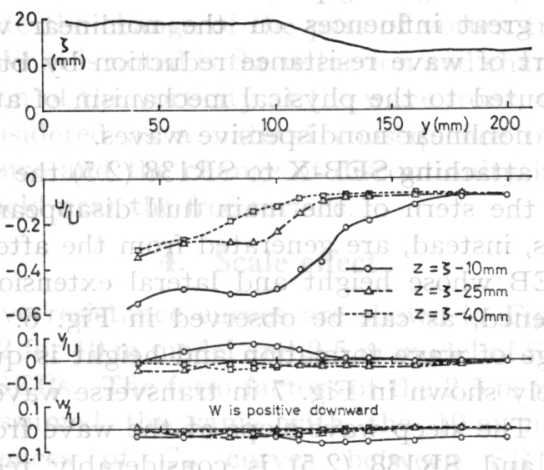

Fig. 4 Measured wave height and disturbance velocities at $x / l=1.16$ of SR138(2.5) at $F n=0.27$ 


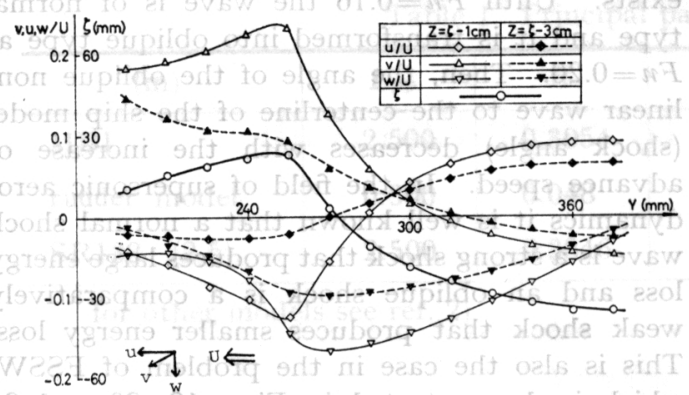

Fig. 5 Measured disturbance velocities at S.S. $7^{7} / 12$ of parabolic wall-sided model WM2 at $F n=0.22(L=2.4 \mathrm{~m})$

wave front, as seen in Fig. 4. The changes of disturbance velocities are also steep where the slope of the wave is. Behind the wave front $u$ is suddenly decreased and $v$ is increased in the flow field near the free surface. These distributions are very similar to those of FSSW around bow of WM2 presented in Fig. 5. Although the shock condition cannot be verified because of the effect of viscous wake, it is obvious that nonlinear waves around the stern have essentially similar characteristics with those around the bow.

\section{Mechanism of resistance reduction}

\subsection{Wave attenuation}

The effect of resistance reduction by $\mathrm{SEB}$ is composed of the decrease of wave pattern resistance $\left(\Delta C_{w p}\right)$ and that of nonlinear wave resistance (approximately $\Delta C_{w}+\Delta C_{w p}$ ). Nonlinear waves called FSSW inevitably occur in the near-field of ships, and they are especially liable to appear at the fore- and aft-ends. Therefore, bulbous bow and SEB which are situated close to the starting point of the nonlinear waves have great influences on the nonlinear waves. A part of wave resistance reduction by bulbs is attributed to the physical mechanism of attenuating nonlinear nondispersive waves.

By attaching SEB-X to SR138 (2.5) the waves from the stern of the main hull disappear, and waves, instead, are generated from the after end of SEB whose height and lateral extension are weakened, as can be observed in Fig. 6. This change of wave formation and height is quantitatively shown in Fig. 7 in transverse wave profiles. The steep wave slope at the wave front of TK1 and SR138 (2.5) is considerably reduced by SEB.

\subsection{Pressure distribution on hull surface}

The resistance reduction by SEB must be re-
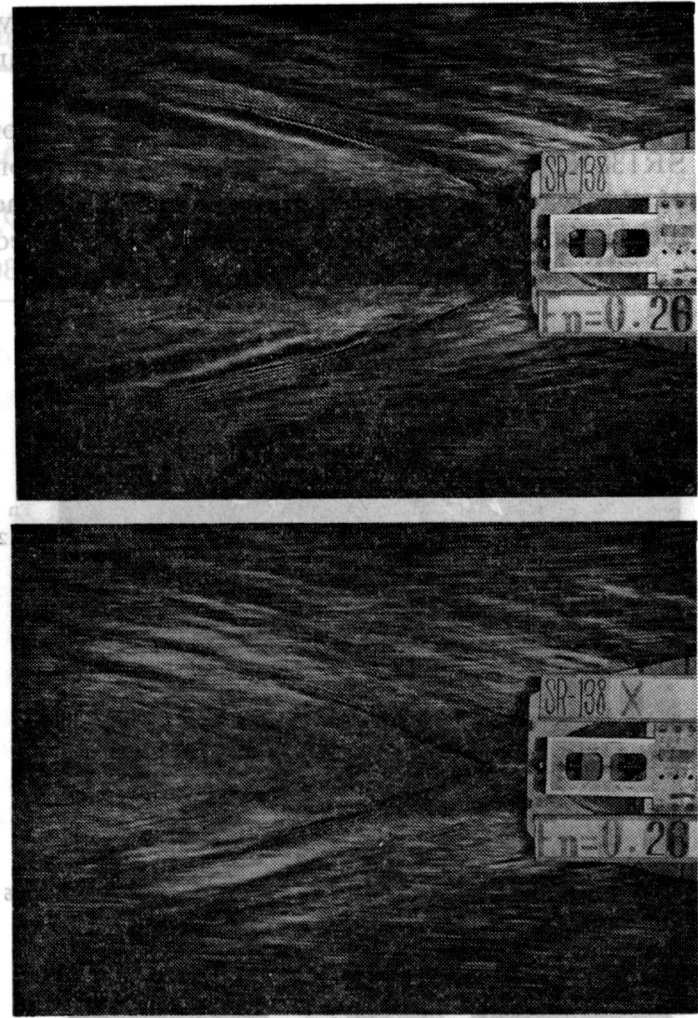

Fig. 6 Difference of stern wave formation between SR138 (2.5) and SR138X(2.5)

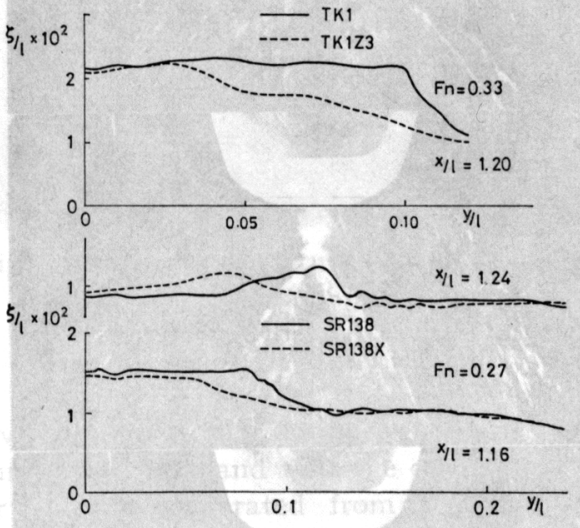

Fig. 7 Transverse profile of stern wave

vealed as a change of pressure distribution on hull surface. For this purpose static pressures were measured on the hull surface of M45 and M45X that have about 60 pressure holes on the port side, some of which were located above the load water line. The results are illustrated 

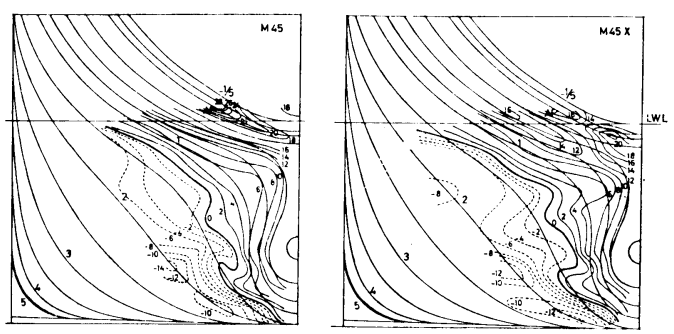

Fig. 8 Change of pressure distribution on hull surface of M45 at $F n=0.27$

in Fig. 3. The region where pressure is high is shifted forward and the form of distribution is quite different around $\mathrm{AP}$ in the neighborhood of the load water line. As a matter of course, this difference of pressure distribution has intimate relation with the difference of wave generation in the vicinity of stern. On the contrary, the difference of wave formation has little to do with the pressure distribution in the deeper region. This implies that the nonlinear waves at the stern is mainly related to the pressure on the hull adjacent to the free surface, which is the reason why SEB is so successful in reducing wave resistance.

\subsection{Momentum loss far behind ship}

The reduction of resistance by SEB can be evaluated by integrating the difference of hull surface pressures. And, at the same time it is evaluated with wave height and momentum loss measured far behind the ship. Suppose that the effect of SEB is limited in the linear dispersive waves, and then the difference of resistance is merely measured as difference of wave height in the far-field. However, if it is also in the nonlinear nondispersive waves such as FSSWs, it will be revealed in the difference of momentum loss far behind the ship, which is surely recorded with SR138 and SR138X as is shown in Fig. 9. The measured momentum loss in the flow-field near the free surface is remarkably reduced by SEB. The difference of resistance obtained by the integration of the difference of pressure distribution is about $20 \mathrm{~g}$, which grossly agrees with $\Delta C_{w}-\Delta C_{w p}$ of SR138X (2.5). This means that SEB effectively reduces nonlinear waves which do not propagate dispersively but dissipate into momentum loss far behind.

\subsection{Vorticity distribution}

Vorticity distributions were measured with TK1 and TK1Z3 at the Ship Research Institute. The flow field behind ships is full of vorticity and the distribution is rather complex, as seen in Fig. 10. The strong vorticity around $Y / l=$

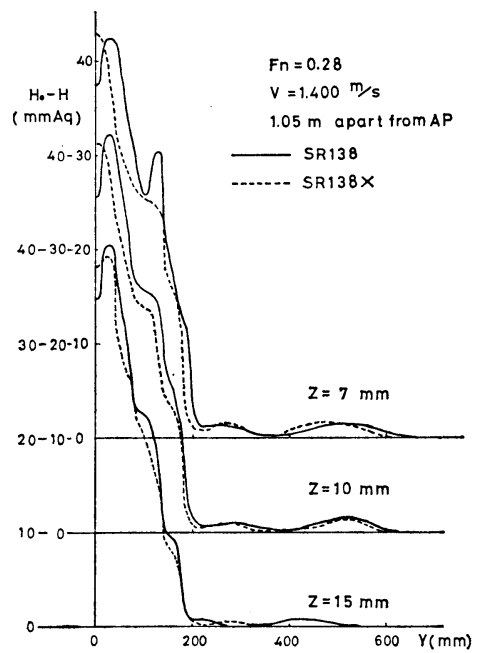

Fig. 9 Momentum loss measured far behind SR138(2.5) and SR138X(2.5)

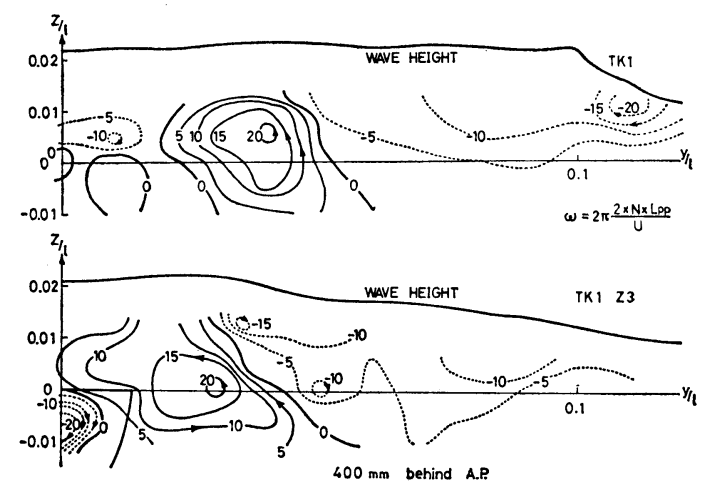

Fig. 10 Vorticity distribution behind TK1 and TK1Z3

0.04 is shifted inward by attaching SEB, which indicate the change of the pass of vortex traveling. What is noted is the reduction and change of location of the vorticity at the wave front, which is considered as a natural consequence of the existence and the change of discontinuity in the flow field near the free surface.

\section{Scale effect}

Wave resistance curves are shown in Figs. 11 and 12 for $10 \mathrm{~m}$ model and $2.5 \mathrm{~m}$ model of SR138 with SEBs. The form factors of the $2.5 \mathrm{~m}$ model are assumed the same with the $10 \mathrm{~m}$ model. Differences of $C_{w}$ curves between SR138X, SR138X1 and SR138X2 are clear on the $10 \mathrm{~m}$ model, although they are not on the $2.5 \mathrm{~m}$ model in the range of $F n>0.23$. The rate of wave 


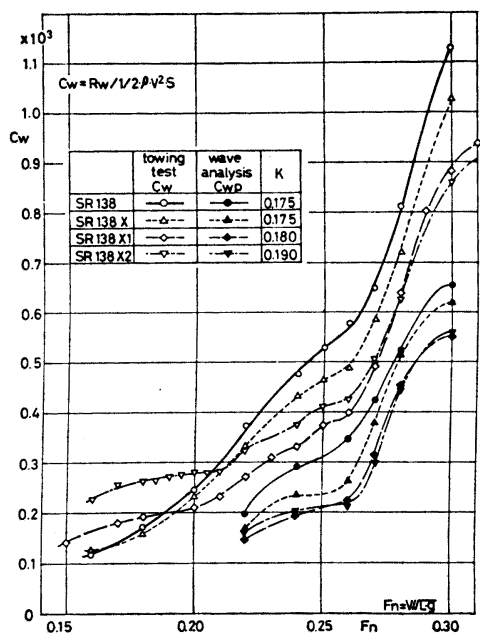

Fig. 11 Wave resistance curve of SR138(10)

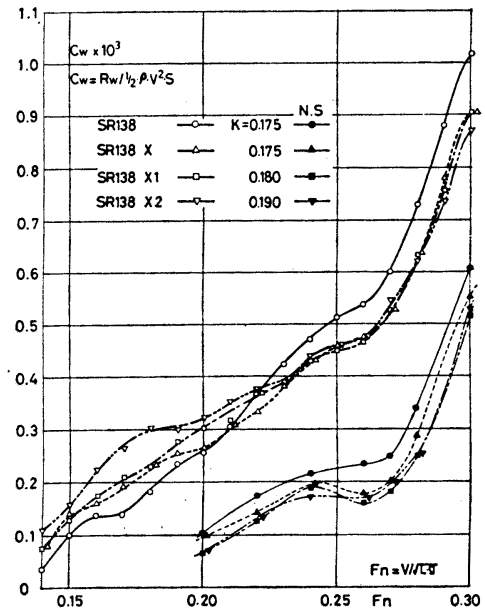

Fig. 12 Wave resistance curve of SR138(2.5)

reduction by SEB-X1 is $25 \%$ on $10 \mathrm{~m}$ model and it is only $12.5 \%$ on $2.5 \mathrm{~m}$ model at the designed Froude number 0.27 . The reduction of wave pattern resistance $\left(C_{w p}\right)$ analysed from longitudinal wave records is about $28 \%$ at $F n=$ 0.27 on both models, though the absolute values of $C_{w p}$ are quite different between the two models.

Although Froude's law of similarity is approximately maintained, wave resistance suffers scale effect by the viscosity of water. Differences of wave contours between $2.5 \mathrm{~m}$ and $10 \mathrm{~m}$ models of SR138 are shown in Figs. 14 and 15. The scale effect is most evident in the heights of waves from the stern. When wave resistance can be divided into those due to bow waves and

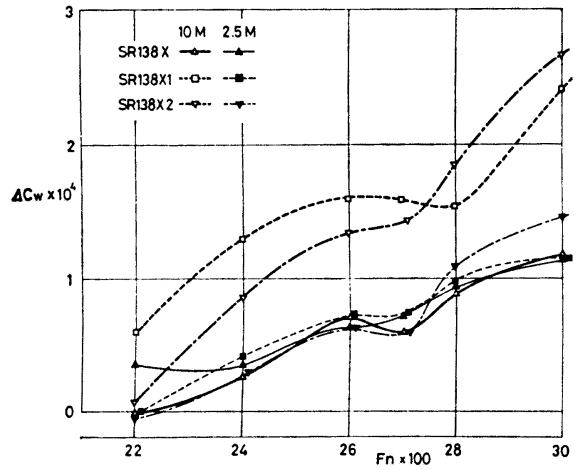

Fig. 13 Scale effect in wave resistance reduction

stern waves, the latter component is relatively small on smaller models, which leads to the smaller rate of resistance reduction by SEB on smaller models. In fact rate of the reduction of wave resistance is small on $2.5 \mathrm{~m}$ model and it is quite enlarged on $10 \mathrm{~m}$ model as is recognized from Figs. 11, 12, and 13.

Measured amplitude functions are shown in Fig. 16 which shows reasonable difference between the geosim models. The scale effect in the reduction of linear wave resistance can be understood as mentioned above.

The resistance reduction also consists in the attenuation of nonlinear waves at the stern. The formation of nonlinear waves does not differ between geosim models or between a model and a full-scale ship, which is certified with the wave patterns of SR138 and TK1. However, the difference of the behavior of water at the front of nonlinear waves is apparently great. One can easily recognize this fact by comparing Fig. 3 with Fig. 6 for the case of SR138 and Fig. 20 with Fig. 21 for the case of TK1, though one has to take notice that Figs. 6 and 20 were photographed with flash-lights whose period of exposure is about $1 / 1000$ second. The nonlinear waves are accompanied with white bubbling on large models. This phenomenon may be partly due to the difference of the absolute pressure in the fluid. The behavior of the free surface which shows that air is drawn into water will be influenced by the absolute pressure in a little similar manner with the problem of cavitation. However, the essential role may be played by viscosity. Some reasoning can be derived from the following experimental results.

The measured wave contours of SR138 on two conditions in Figs. 14 and 15 show that the waves from the forebody also suffer scale effect, that is, waves are higher on a larger model. 


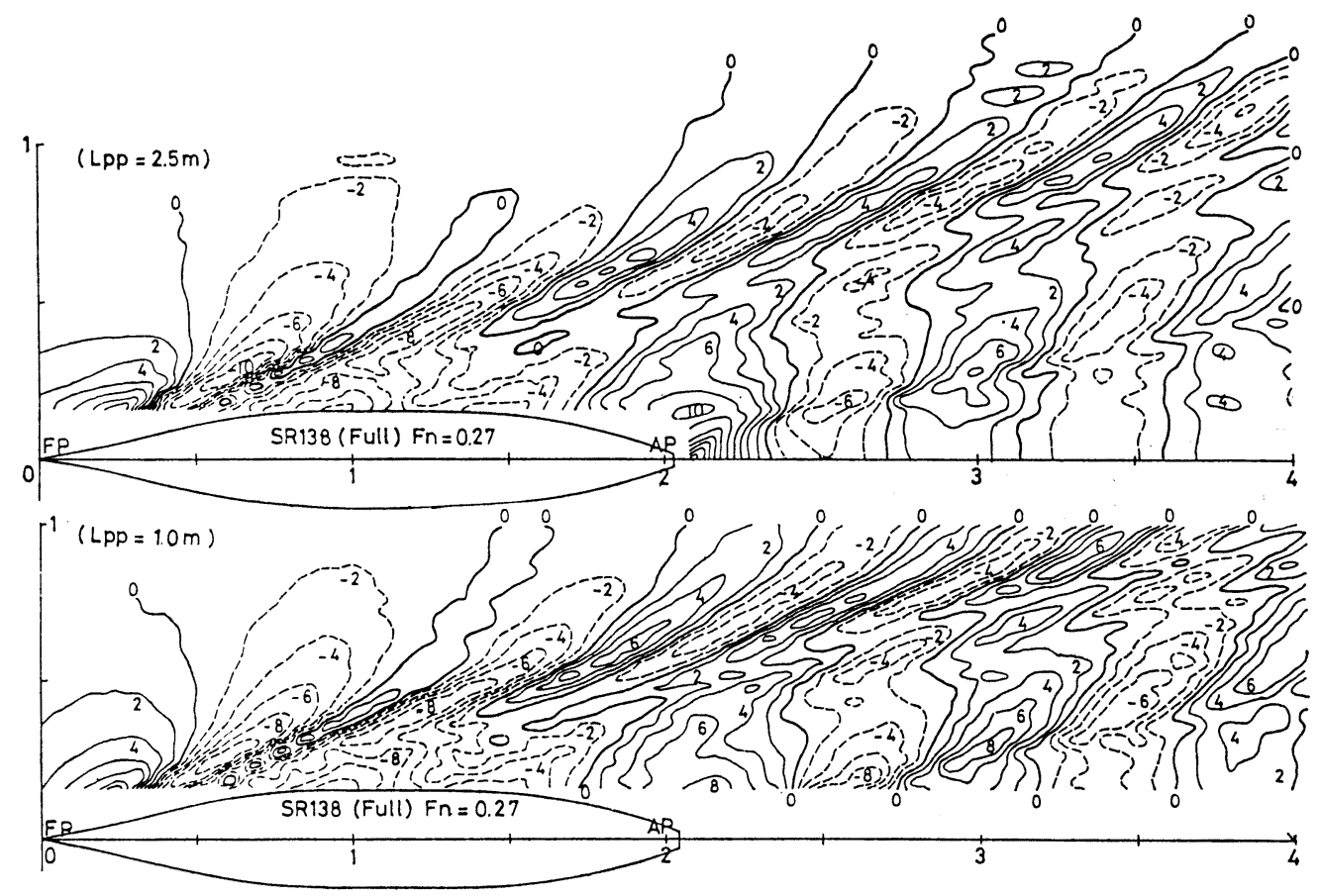

Fig. 14 Comparison of wave contours between $10 \mathrm{~m}$ and $2.5 \mathrm{~m}$ models (full load condition) (wave height in $2 \zeta / L \times 1000$ )

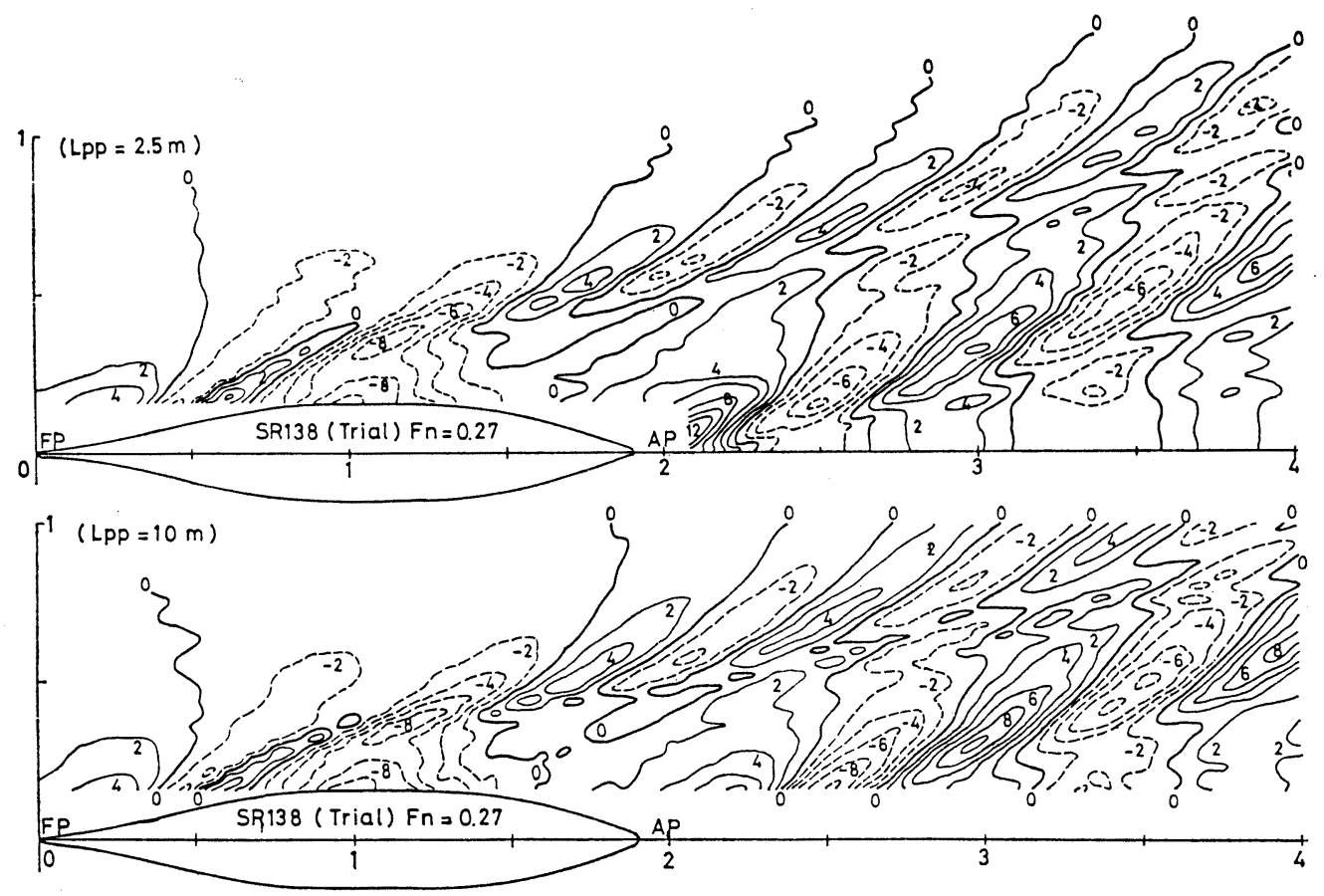

Fig. 15 Comparison of wave contours between $10 \mathrm{~m}$ and $2.5 \mathrm{~m}$ models (trial condition) 
This is more clearly seen in Fig. 17 which is a case of SR138 and SR138X. This phenomenon has already been noticed in the Research Program SR138 of the Shipbuilding Research Association of Japan. Nonlinear waves are generated in the near-field around bow and stern, and they produce a steep slope of free surface on the front and turbulence of free surface behind the front. In general, there is possibility for viscosity

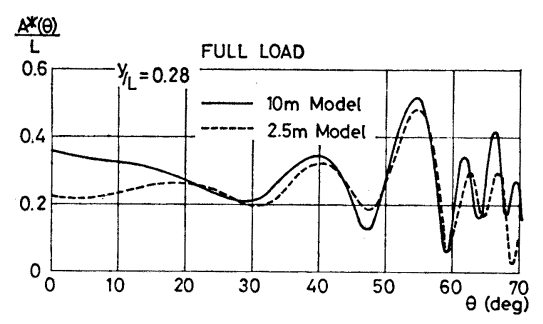

Fig. 16 Weighted amplitude functions of $\mathrm{SR} 138$ at $F n=0.27$
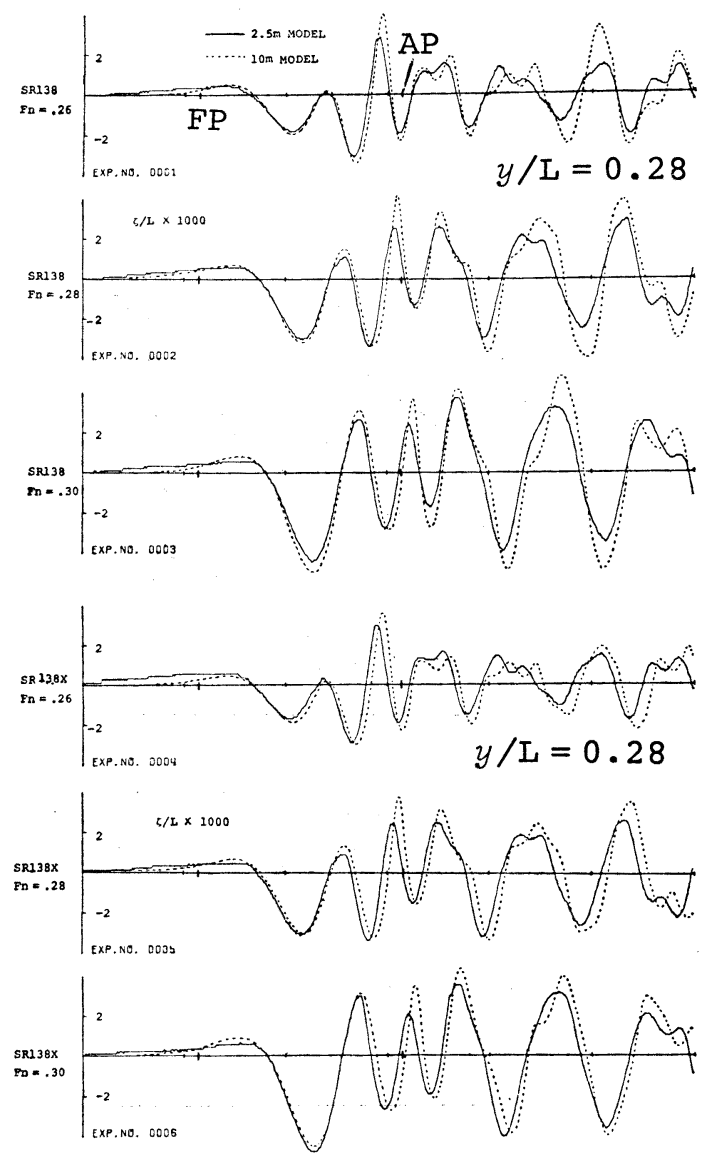

Fig. 17 Comparison of longitudinal wave profiles of geosim models to play a certain role in the properties of this kind of nonlinear wave. It is supposed that the steep nonlinear waves that constitute lines of discontinuity and are accompanied by unsteady turbulent free surface will suffer a kind of diffusive effect due to viscosity and that the scale effect in the wave height of bow waves will be attributed to the imaginary viscosity on the free surface. If this assumption is firmly verified through advanced thorough studies, it is considered that there are two ways of viscosity to attenuate free surface waves; viscous effect on hull surface and on free surface. Thus, the scale effect in the reduction of wave resistance by SEB will be mostly attributed to the two effects of viscosity.

\section{Improved stern-end-bulb for a passenger boat}

SEB-Z3 was very effective and the saving ratio of EHP reached up to more than 5\%. However, the wave pattern picture of TK1Z3 still shows conspicuous nonlinear stern waves, which indicates the possibility of further improvement. Some new kinds of SEBs are designed for TK1 in order to learn how far resistance can be reduced by SEB. The length of SEB-Z3 is limited by the expected unfavorable effect in maneuverability at a harbor, and simultaneously it requires certain volume. This resulted in the round-shaped end of SEB shown in Fig. 18, which cannot be the optimal configuration of SEB. The newly designed SEBs are $Z 6$ and $Z 7$ whose length and volume are both increased. $\mathrm{Z4}$ is one whose length is, on the contrary, shortened.

The results of towing test are shown in the form of required towing force in Fig. 19. The reduction of total resistance by SEB-Z7 is about $3.7 \%$ at $F n=0.33$, while that by SEB-Z3 is about $2.8 \%$. The estimated resistance reduc-

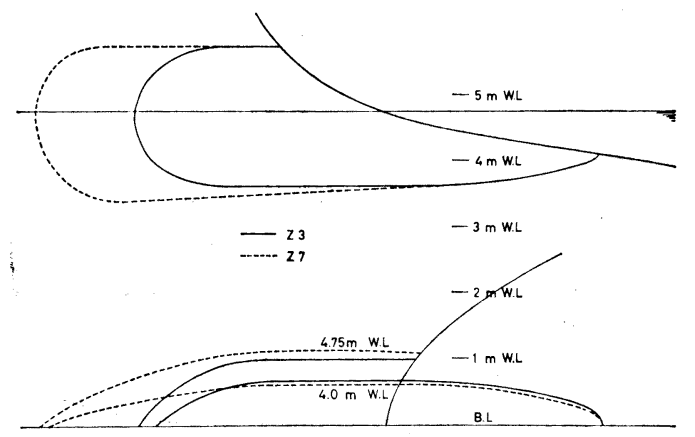

Fig. 18 Configuration of SEB-Z3 and Z7 for TK1 


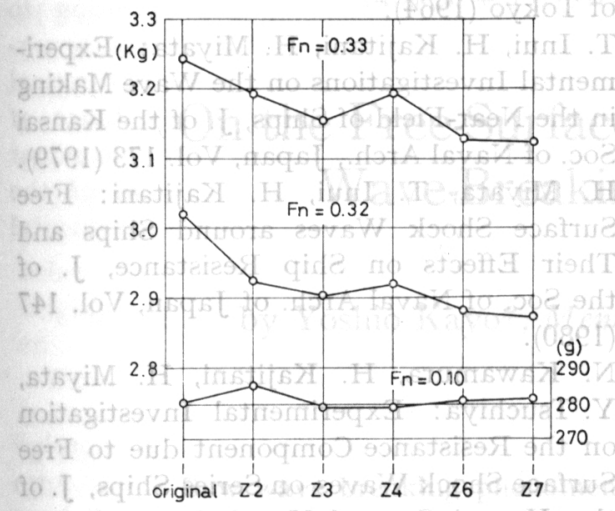

Fig. 19 Comparison of total resistance of TK1

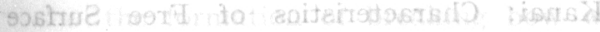

tion on a full-scale ship can reach up to $7 \%$, when miscellaneous limitations are removed during the course of designing SEB. It is noted that the resistance increase at a very low Froude number due to the attachment of SEB is rather small in comparison with the increase of the wetted surface area.

The comparison of wave patterns around the stern of TK1 with and without SEB is shown in Fig. 20. The attenuation of the wave height and the reduction of the lateral extension of the stern nonlinear wave system are clearly observed. On a full-scale ship the wedge-shaped region enclosed by the two lines of wave front of the nonlinear stern waves looks white with air draw as is seen in Fig. 21, which is a consequence of the unsteadiness of the free surface. This region will be reduced by SEB.

\section{Conclusion}

Waves around the stern are most complicated and require further detailed experimental and theoretical investigations. At the experimental tank of the University of Tokyo, fundamental

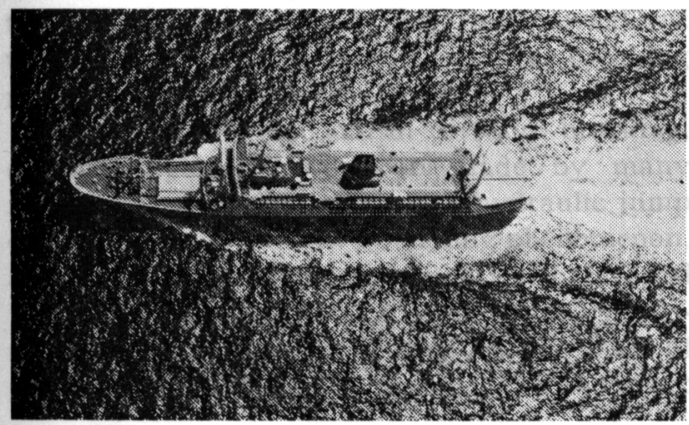

Fig. 21 Picture of waves around full-scale ship of TK1
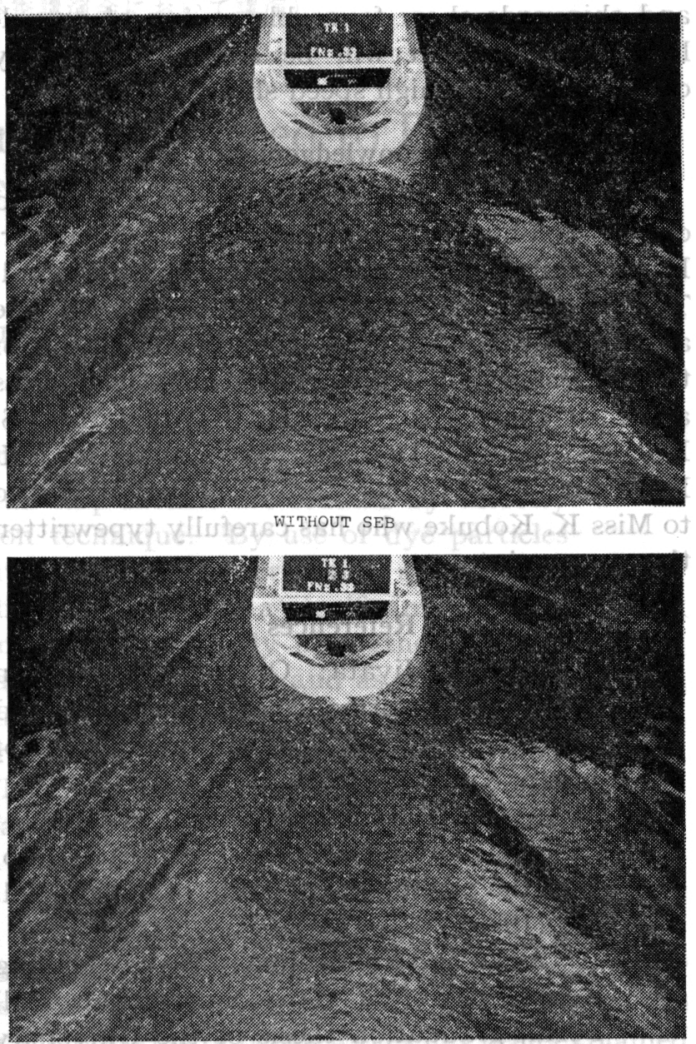

WITH SEB-Z3

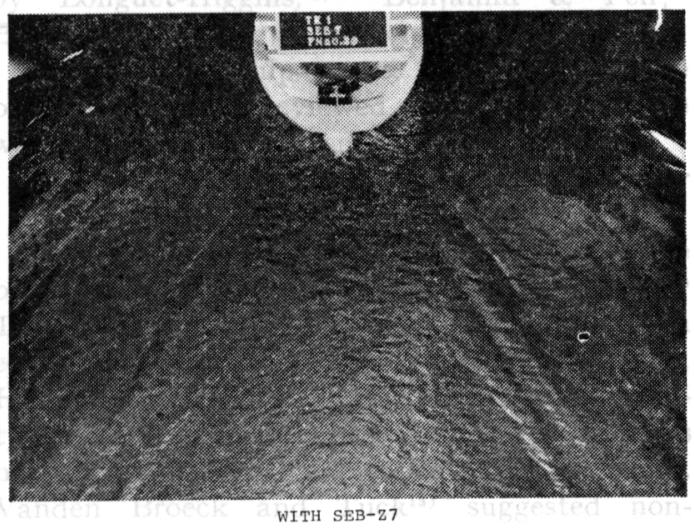

Fig. 20 Stern wave pictures of TK1 at $F n=0.33$

investigations into the waves around the stern are underway with simple hull forms, which will be reported in the near future.

It is decided by the owner of SUTORECHIAMARU (model name TK1) that she will be equipped with a SEB similar to $Z 3$ in January 1981 as the first ship with a SEB. Almost all the model ships (more than 10) served for experiments with SEBs at the basins of our university 
and shipyards show favorable saving of propelling power, and it will be adopted by many other middle and high speed ships.

\section{Acknowledgement}

Some parts of the experiments were carried out at the Ship Research Institute of Japan. Dr. H. Adachi's collaboration is highly appreciated. This research is, as usual, supported by the authors' colleagues at the experimental tank of the University of Tokyo. The authors express a lot of thanks to Prof. H. Kajitani, Mr. S. Kuzumi, Mr. Y. Doi, Mr. M. Kanai, Mr. M. Nito, Mr. T. S. Chong, Mr. M. Takai and also to Miss K. Kobuke who has carefully typewritten the manuscript.

\section{References}

1) T. Inui, H. Miyata: On the Optimization of Overall Performance of Rudders (Second Report), J. of the Soc. of Naval Arch. of Japan, Vol. 145 (1979).

2) H. Miyata, Y. Tsuchiya, T. Inui, H. Adachi: Resistance reduction by Stern-End-Bulb, J. of the Soc. of Naval Arch. of Japan, Vol. 148 (1980).

3) T. Arii, S. Fukushima: Wave Making Resistance of a Two-Dimensional Hull Form, graduation thesis of the University of Tokyo (1964).

4) T. Inui, H. Kajitani, H. Miyata: Experimental Investigations on the Wave Making in the Near-Field of Ships, J. of the Kansai Soc. of Naval Arch., Japan, Vol. 173 (1979).

5) H. Miyata, T. Inui, H. Kajitani: Free Surface Shock Waves around Ships and Their Effects on Ship Resistance, J. of the Soc. of Naval Arch. of Japan, Vol. 147 (1980).

6) N. Kawamura, H. Kajitani, H. Miyata, Y. Tsuchiya: Experimental Investigation on the Resistance Component due to Free Surface Shock Waves on Series Ships, J. of the Kansai Soc. of Naval Arch., Japan, Vol. 179 (1980).

7) M. Takahashi, H. Kajitani, H. Miyata, M. Kanai: Characteristics of Free Surface Shock Waves around Wedge Models, J. of the Soc. of Naval Arch. of Japan, Vol. 148 (1980).

8) H. Miyata: Characteristics of Nonlinear Waves in the Near-Field of Ships and Their Effects on Resistance, Proc. of 13th Symposium on Naval Hydrodynamics, Tokyo (1980).

(All except 1) and 3) are written in English) 